\title{
Estrutura populacional e distribuição espacial de Miconia prasina D.C. (Melastomataceae) em duas áreas de Floresta Atlântica na Ilha Grande, RJ, Sudeste do Brasil
}

\author{
Rafaela Dias Antonini ${ }^{1}$ e André Felippe Nunes-Freitas ${ }^{1,2,3}$
}

Recebido em 12/09/2003. Aceito em 28/02/2004

\begin{abstract}
RESUMO - (Estrutura populacional e distribuição espacial de Miconia prasina D.C. (Melastomataceae) em duas áreas de Floresta Atlântica na Ilha Grande, RJ, Sudeste do Brasil). O objetivo deste estudo foi identificar o padrão de distribuição espacial e a estrutura populacional de Miconia prasina D.C. em duas áreas de Floresta Atlântica na Ilha Grande, RJ. A área I (AI) é uma floresta secundária em regeneração a cerca de 30 anos, enquanto a área II (AII) é uma floresta primária, com poucas evidências de ação antrópica. Em cada área foram estabelecidas 40 parcelas $\left(25 \mathrm{~m}^{2}\right)$, onde registrou-se o número de indivíduos e foram medidos a altura e o DAP de cada indivíduo. Determinou-se o padrão de distribuição espacial através do índice de Dispersão de Morisita (Id). Testou-se possíveis diferenças na abundância, DAP e altura entre as duas populações através do Teste T de Student. O índice de dispersão foi de 1,91 na AI e de 3,32 na AII, indicando um padrão agregado nas duas áreas. Não houve diferença significativa entre as abundâncias das duas populações. O DAP e a altura das duas populações diferiram significativamente, com a AII apresentando valores médios mais baixos que a AI. Os dados sugerem que a população da AI é uma população em estado mais avançado de ocupação do habitat do que a população da AII, já que apresenta médias de DAP e altura mais altas.
\end{abstract}

Palavras-chave: Miconia prasina, estrutura etária, densidade populacional

\begin{abstract}
Population structure and spatial distribution of Miconia prasina D.C. (Melastomataceae) in an Atlantic Rainforest area at Ilha Grande, RJ, Southeastern Brazil). The aim of this study was to identify the spatial distribution pattern and the populational structure of Miconia prasina D.C. in two Atlantic Forest areas at Ilha Grande, RJ. The area I (AI) is a secondary forest near 30 years of regeneration, and area II (AII) is a primary forest with few evidences of anthropic action. On each area, 40 plots $\left(25 \mathrm{~m}^{2}\right)$ were established, where the number of individuals, height and the BHD of each individual were recorded. The pattern of spatial distribution was determined using the Morisita's Dispersion Index (Id). Possible differences on abundance, BHD and height between the two populations were tested using Student's T Test. The Morisita's Index value was 1.91 in AI and 3.32 in AII, indicating a clumped distribution pattern on both areas. There was no significant difference in abundance between populations. BHD and tree height differed significantly between populations, with AII presenting lower mean values than AI. Data suggest that the AI population is on a more advanced level of habitat occupation than AII, since it has greater mean values of BHD and height.
\end{abstract}

Key words: Miconia prasina, age structure, population density

\section{Introdução}

Atualmente, uma das maiores preocupações para a conservação das florestas tropicais é a transformação e exploração deste habitat (Rossi \& Huguchi 1998). A exploração modifica severamente a estrutura do habitat (Lieberman \& Dock 1982; Hoffman 1998), atuando diretamente no padrão de distribuição espacial (Almeida et al. 1998) e fenológico das espécies nativas (Clark \& Clark 1987; Costa \& Magnusson 2003), já que diferentes fatores do ambiente, como luz incidente, umidade e disponibilidade de nutrientes também serão afetados (Rossi \& Huguchi 1998). Por outro lado, a alteração de ambientes naturais permite a entrada de novas espécies nestes ambientes, principalmente espécies que possuam uma grande resistência a condições ambientais mais extremas e que podem ser consideradas espécies pioneiras ou invasoras. Essas plantas apresentam como atributo único a capacidade de se desenvolver em condições ambientais não muito favoráveis para a sobrevivência de outros vegetais (Roy 1990).

A família Melastomataceae possui mais de 4.800 espécies, sendo a maioria de distribuição Neotropical

\footnotetext{
Setor de Ecologia, Instituto de Biologia Roberto Alcântara Gomes - IBRAG, Universidade do Estado do Rio de Janeiro

Programa de Pós-graduação em Biologia, área de Concentração em Ecologia, IBRAG, Universidade do Estado do Rio de Janeiro

3 Autor para correspondência: Setor de Ecologia, Instituto de Biologia Roberto Alcântara Gomes, UERJ. Rua São Francisco Xavier, 524, CEP 20550-011, Maracanã, Rio de Janeiro, RJ, Brasil (afnfreitas@infolink.com.br)
} 
(Stiles \& Rosselli 1983). O gênero Miconia é o maior da família com cerca de 1.000 espécies (Meyer 1998), que podem ser componentes do sub-bosque de florestas primárias, porém ocorrem principalmente em áreas secundárias, bordas de floresta e clareiras naturais no interior de florestas, por esta razão podendo ser consideradas como espécies pioneiras ou invasoras (Schupp et al. 1989; Denslow et al. 1990; Ellison et al. 1993). Em geral, as plantas desse gênero são extremamente diversas em sua arquitetura reprodutiva e vegetativa (Judd 1986), produzem uma grande quantidade de sementes e suas plântulas podem se estabelecer rapidamente no solo de ambientes degradados (Snow 1965; Stiles \& Rosselli 1983).

A Ilha Grande, localizada no município de Angra dos Reis, no litoral sul do Estado do Rio de Janeiro, é um dos maiores fragmentos de Mata Atlântica do Estado, possuindo áreas de floresta em diferentes níveis de regeneração (Cogliatti-Carvalho \& Rocha 2001). Nestas áreas são encontradas diferentes espécies do gênero Miconia, dentre elas Miconia prasina D.C., uma espécie característica de áreas em recomposição (Stiles \& Rosselli 1983; Meyer 1998).

Neste estudo foram analisados a estrutura e o padrão de distribuição espacial de duas populações de Miconia prasina em duas áreas de floresta na Ilha Grande, em diferentes estágios de regeneração. Especificamente, procurou-se responder às seguintes questões: i) Qual é o padrão de distribuição espacial das duas populações de $M$. prasina nas áreas estudadas?; ii) Qual é a estrutura etária das duas populações estudadas?; iii) Há diferença na abundância, altura e diâmetro à altura do peito (DAP) dos indivíduos entre as duas populações?; iv) As diferenças encontradas estão relacionadas ao grau de perturbação das duas áreas?

\section{Material e métodos}

Área de Estudo - O estudo foi realizado em duas áreas de Floresta Atlântica em diferentes estágios de regeneração, próximas à Vila Dois Rios, na Ilha Grande, no Município de Angra dos Reis, sul do Estado do Rio de Janeiro, Sudeste do Brasil. A Ilha Grande possui clima quente e úmido, com temperatura média do ar de $23^{\circ} \mathrm{C}$ e pluviosidade anual de aproximadamente 2.300mm (Araújo \& Oliveira 1988). A cobertura vegetal situa-se no domínio da floresta ombrófila densa (Veloso et al. 1991; Oliveira 2002) com diferentes níveis de regeneração após distúrbios humanos. Aárea I (AI; $23^{\circ} 19^{\prime} 92,3^{\prime}$ 'S e $44^{\circ} 12^{\prime} 04,0^{\prime}$ 'W) é área de
Floresta Atlântica secundária com aproximadamente 30 anos de regeneração após toda a vegetação ter sido retirada para construção de uma represa (Almeida et al. 1998). Esta área está localizada a cerca de $80 \mathrm{~m}$ de altitude, possui o dossel variando entre 15 a $25 \mathrm{~m}$ de altura e diâmetro à altura do peito (DAP) entre $10 \mathrm{e}$ $30 \mathrm{~cm}$. O solo possui grande quantidade de blocos rochosos e estreita camada de folhiço com 0 a $30 \mathrm{~mm}$ de espessura (Almeida et al. 1998). O sub-bosque é praticamente ausente, sendo a flora de epífitas representada por poucas espécies (A.F. Nunes-Freitas, comunicação pessoal), a maioria da família Bromeliaceae (Almeida et al. 1998). A área II (AII; $23^{\circ} 10^{\prime} 58,0^{\prime \prime} \mathrm{S}$ e $44^{\circ} 12^{\prime} 49,9^{\prime}$ 'W), é área com pouca evidência de perturbação antrópica, localizada a $240 \mathrm{~m}$ de altitude. Em AII, a altura do dossel varia de 25 a 30 metros, com abundância de árvores de grande porte (DAP $>50 \mathrm{~cm})$ e sub-bosque pouco denso, apresentando camada de folhiço profunda (50-200mm) e com rica e abundante flora de epífitas (Almeida et al.1998).

Para estudar o padrão de distribuição espacial e a estrutura etária de Miconia prasina D.C., foram demarcadas aleatoriamente 40 parcelas de $25 \mathrm{~m}^{2} \mathrm{em}$ cada uma das áreas estudadas, totalizando 0,1 ha de área amostrada. Em cada parcela registrou-se o número de indivíduos de $M$. prasina, a altura e o DAP de cada um dos indivíduos amostrados. Para avaliar o padrão de distribuição espacial da espécie, utilizou-se o Índice de Dispersão de Morisita (Id) (Brower \& Zar 1984):

$$
I_{d}=n \quad\left[\frac{\sum x_{i}^{2}-\sum x_{i}}{\left(\Sigma x_{i}\right)^{2}-\Sigma x_{i}}\right]
$$

onde $\mathrm{n}$ é o número de parcelas amostradas e $\mathrm{x}_{\mathrm{i}} \mathrm{o}$ número de indivíduos em cada parcela amostrada (Brower \& Zar 1984). A significância do Índice de Dispersão de Morisita $\left(I_{d} \neq 1\right)$ foi testada através do teste $\mathrm{F}$ para significância do $\mathrm{I}_{\mathrm{d}}(\mathrm{gl}=\mathrm{n}-1 ; \mathrm{p}<0,05)$ (Poole 1974):

$$
\mathrm{F}=\frac{\mathrm{I}_{\mathrm{d}}(\mathrm{N}-1)+\mathrm{n}-\mathrm{N}}{\mathrm{n}-1}
$$

onde $I_{d}$ é o valor calculado do Índice de Dispersão de Morisita, $\mathrm{n}$ o número de parcelas e $\mathrm{N}$ o número total de indivíduos encontrados em todas as $\mathrm{n}$ parcelas. $\mathrm{O}$ valor calculado de $\mathrm{F}$ é comparado com o valor da tabela de F, com n - 1 graus de liberdade para o numerador e infinito $(\infty)$ para o denominador.

Realizou-se a análise comparativa das variáveis altura e DAP das duas populações estudadas através de gráficos de barras com os valores encontrados para as duas populações. Posteriormente, testou-se possíveis 
diferenças na estrutura das duas populações estudadas utilizando o Teste $\mathrm{T}$ de Student entre os valores de abundância de indivíduos, DAP e altura das duas populações de M. prasina (Zar 1999).

Como áreas com tempos de regeneração diferentes apresentam estruturas distintas, as condições de penetração de luz tendem a ser diferenciadas, especialmente devido às diferenças na densidade do dossel (Chazdon \& Fetcher 1984; Denslow 1987; MacDougal \& Kellman 1992; Canham et al. 1994; Messier 1996; Clark et al. 1996; Svenning 2000). Com base nesta sugestão, foram relacionados indiretamente a intensidade média de luz em cada uma das áreas com os dados de tamanho populacional e estrutura etária. Os dados de intensidade média de luz aportando ao nível do solo para as duas áreas estudadas foram obtidos em Almeida et al. (1998), que realizaram medidas mensais da intensidade de luz ao longo de 12 meses. Para maiores detalhes sobre a metodologia, veja Almeida et al. (1998).

\section{Resultados e discussão}

Foram amostrados o total de 141 indivíduos de M. prasina nas duas áreas, sendo $80 \mathrm{em} \mathrm{AI} \mathrm{e} 61 \mathrm{em}$ AII (Tab. 1). Os valores do Índice de Dispersão de Morisita (Id) encontrados para as duas populações de M. prasina foram de $1,91(\mathrm{~F}=2,843 ; \mathrm{p}<0,05) \mathrm{em}$ AI e de 3,32 ( F = 7,118; p < 0,05) em AII (Tab. 1), indicando um padrão de distribuição agregado para as duas populações (Id > 1; Brower \& Zar 1984). Um padrão agregado de distribuição é característico de espécies vegetais dispersas por animais ou que realizam sua dispersão por autocoria (barocoria) e está relacionado com a quantidade de sementes produzidas e a duração do período de frutificação (Janzen 1976).

A estratégia de dispersão mais freqüentemente observada e descrita nas espécies da família

Tabela 1. Estrutura etária de duas populações de Miconia prasina D.C. estudadas em áreas de Floresta Atlântica da Ilha Grande, RJ. $\mathrm{AI}=$ área de Floresta Atlântica com aproximadamente 30 anos de regeneração; $\mathrm{AII}$ = área de Floresta Atlântica com pouca evidência de perturbação; Id = Índice de Dispersão de Morisita; $\mathrm{N}=$ abundância; $\mathrm{H}=$ altura (em metros); $\mathrm{DAP}=$ diâmetro à altura do peito (em centímetros). Letras diferentes indicam diferenças significativas no Teste $\mathrm{T}$ de Student $(\mathrm{p}<0,05)$.

\begin{tabular}{ccccc}
\hline Área & Id & $\mathrm{N}$ & $\mathrm{H}$ & $\mathrm{DAP}$ \\
\hline AI & 1,91 & $80^{\mathrm{a}}$ & $3,93 \pm 3,06^{\mathrm{a}}$ & $5,43 \pm 6,57^{\mathrm{a}}$ \\
AII & 3,32 & $61^{\mathrm{a}}$ & $1,63 \pm 1,48^{\mathrm{b}}$ & $2,48 \pm 3,18^{\mathrm{b}}$ \\
\hline
\end{tabular}

Melastomataceae é a zoocoria (Levey 1990). As espécies dessa família são recurso importante para diferentes populações animais das matas secundárias (Stiles \& Rosselli 1983; Levey 1988; Barnea et al. 1992; Galetti \& Stotz 1996; Poulin et al. 1999), em geral apresentando longo período de frutificação e oferecendo grande quantidade de frutos ao longo desse período (Stiles \& Rosselli 1983; Levey 1990; Barnea et al. 1992; Galetti \& Stotz 1996; Poulin et al. 1999). Na Ilha Grande, M. prasina tem período relativamente longo de frutificação (aproximadamente seis meses) e com frutos em diferentes estágios de maturação ao longo deste período (observação pessoal). Quando maduros, seus frutos são arroxeados e pequenos e atraem grande variedade de dispersores, principalmente aves, como citado para diversas espécies da família Melastomataceae (Stiles \& Rosselli 1983; Galetti \& Stotz 1996; Meyer 1998; Poulin et al.1999; Pereira \& Mantovani 2001).

No entanto, apesar da elevada taxa de visitação em $M$. prasina pela avifauna local, possivelmente a síndrome de dispersão mais freqüente para esta espécie seja a autocoria (barocoria) ou esta em associação com a zoocoria, como sugerido para M. cinnamomifolia (Pereira \& Mantovani 2001). Em ambos os casos, grande parte dos frutos e sementes seria depositada próximo à planta-mãe naturalmente ou pelos seus dispersores. Assim, como M. prasina produz grande número de frutos ao longo do seu período de frutificação, parte destes frutos não seria utilizada como recurso por seus dispersores, sendo depositados no solo naturalmente por abscisão. Conjuntamente, com as aves forrageando nos indivíduos de $M$. prasina, também haveria alta taxa de abscisão de frutos sobre a copa da planta-mãe (Pereira \& Mantovani 2001).

Os valores obtidos também indicaram que a população de AII apresentou padrão de agregação maior que o da AI (Tab. 1), o que pode estar relacionado à estrutura das áreas. O fato da população de M. prasina de AII apresentar valor de $I d$ mais elevado do que o de AI pode estar relacionado às condições estruturais da vegetação e, possivelmente, à incidência luminosa que aporta no ambiente. Em florestas primárias, as espécies do gênero Miconia vão estar associadas principalmente a áreas de clareiras naturais ou bordas de matas (Schupp et al. 1989; Denslow et al. 1990; Ellison et al. 1993), nas quais a taxa de incidência de luz é maior do que em áreas no interior da mata. AII é área com pouca evidência de perturbação antrópica, onde a quantidade de luz aportando sobre o nível do solo é baixa, equivalendo a $1,3 \%$ da 
luminosidade que atinge o dossel (Almeida et al. 1998). Nesta área, a maior penetração de luz se dá nos locais onde ocorrem clareiras naturais (formadas pela queda de galhos e/ou árvores) ou bordas naturais (próximas aos rios), assim como é sugerido para outras áreas de florestas tropicais (Chazdon \& Fetcher 1984; Denslow 1987; MacDougal \& Kellman 1992; Clark et al. 1996; Svenning 2000). Em AI, que apresenta maior abertura do dossel por ser uma área em recomposição, há distribuição mais homogênea de luz aportando sobre o solo, equivalente a $3,8 \%$ daquela que atinge o dossel (Almeida et al. 1998), pouco mais do que o dobro da incidência ao nível do solo de AII, o que pode explicar o menor grau de agregação.

As duas populações estudadas apresentaram o maior número de indivíduos concentrado nas classes inferiores de altura (Fig. 1) e de DAP (Fig. 2), com poucos indivíduos nas classes de maior tamanho, indicando que ambas as populações estão em crescimento (Martins 1993). Assim, os indivíduos com maior DAP seriam aqueles reprodutivamente maduros e potencialmente capazes de manter o estoque de sementes na área, e indivíduos jovens garantiriam a manutenção da população, mesmo havendo alta taxa de mortalidade destes indivíduos até o seu estabelecimento (Dislich \& Mantovani 1998).

Não houve diferença significativa entre as abundâncias das duas populações (Tab. $1 ; \mathrm{t}=-1,398$; $\mathrm{gl}=70 ; \mathrm{p}=0,166)$. Apesar do tamanho populacional de $M$. prasina não ter diferido significativamente entre as duas áreas estudadas, houve diferença na estrutura tanto para altura $(\mathrm{t}=-5,397 ; \mathrm{gl}=107 ; \mathrm{p}<0,001)$ quanto para DAP $(\mathrm{t}=-3,750 ; \mathrm{gl}=102 ; \mathrm{p}<0,001)($ Tab. 1$) . \mathrm{A}$ população de AI apresentou indivíduos com classes

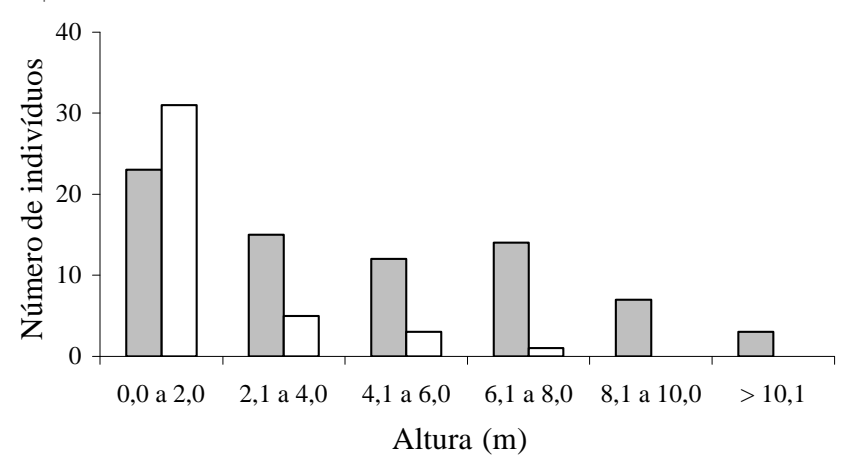

Figura 1. Distribuição das duas populações de Miconia prasina D.C. em classes de altura (em metros) estudadas em áreas de Floresta Atlântica da Ilha Grande, RJ. AI = área de Floresta Atlântica com aproximadamente 30 anos de regeneração $(n=80)$; AII = área de Floresta Atlântica com pouca evidência de perturbação $(\mathrm{n}=61)$. $\square=\mathrm{AI}, \square=$ AII.

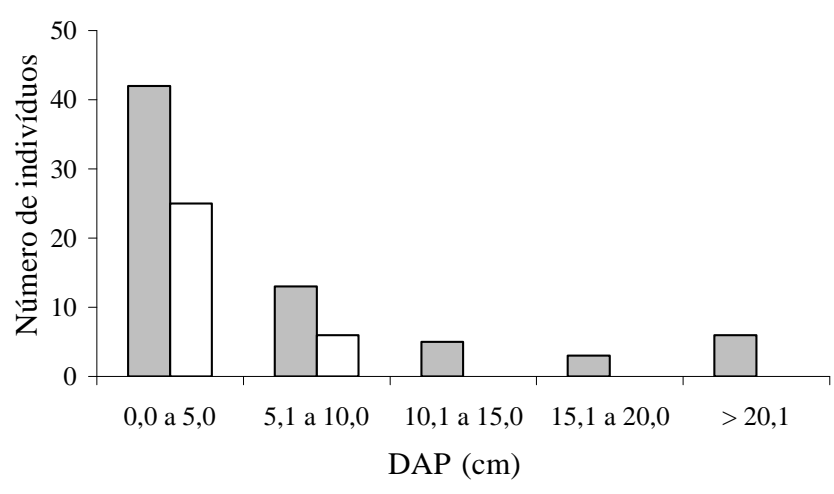

Figura 2. Distribuição das duas populações de Miconia prasina D.C. em classes de diâmetro à altura do peito (DAP) (em centímetros) estudadas em áreas de Floresta Atlântica da Ilha Grande, RJ. AI = área de Floresta Atlântica com aproximadamente 30 anos de regeneração $(\mathrm{n}=80)$; AII = área de Floresta Atlântica com pouca evidência de perturbação $(n=61) . \square=$ AI, $\square=$ AII.

de altura e de DAP mais elevados, enquanto em AII os indivíduos possuíam no máximo $8 \mathrm{~m}$ de altura (Fig. 1) e 10cm de DAP (Fig. 2). Como, de forma geral, o diâmetro do tronco guarda certa proporcionalidade com a idade da árvore (Martins 1993) é possível afirmar que populações com classes de DAP mais elevados estão em estágio de sucessão mais avançado do que as com menor DAP. Com base nos valores de DAP encontrados para a população de $M$. prasina de AI e o fato destes serem significativamente maiores que os da AII, pode-se dizer que a população da AI encontra-se em estágio sucessional mais avançado que a da AII, o que pode estar relacionado à estrutura da vegetação e ao estágio de sucessão das duas áreas.

Áreas de floresta tropical apresentam baixo percentual de penetração de luz (Chazdon \& Fetcher 1984; Denslow 1987; MacDougal \& Kellman 1992; Clark et al. 1996; Svenning 2000), havendo diferenças no grau de penetração entre áreas distintas devido a diferenças na estrutura (p.ex., altura e abertura do dossel, densidade de árvores) e no grau de perturbação das áreas. Além disso, é sugerido que as plantas de florestas tropicais são freqüientemente limitadas pela luz, sejam elas espécies tolerantes à sombra ou espécies pioneiras (Chazdon 1986; Denslow 1987; Welden et al. 1991; Oberbauer et al. 1993; Ellison et al. 1993; Cunningham 1997; Amézquita 1998). Como a AI é uma área em regeneração e a AII sofreu apenas corte seletivo, as duas áreas apresentam estruturas e regimes de incidência de luz distintos (Almeida et al. 1998), com a AI apresentando incidência maior e mais homogênea de luz do que a AII (Almeida et al. 1998). Na AII, a população apenas receberia a entrada de novos indivíduos quando houvesse condições 
favoráveis para a germinação das sementes, isto é, quando ocorresse a formação de clareiras e aumento da incidência luminosa. Desta forma, as duas populações de $M$. prasina responderiam de forma diferenciada, apresentando idades diferentes, sendo a da AII mais nova por estar possivelmente associada a clareiras naturais do interior da mata.

Nossos dados permitem concluir que o padrão de distribuição agregado encontrado para as duas populações de $M$. prasina pode estar relacionado tanto à estratégia de dispersão barocórica (e à associação desta com a zoocoria), quanto com a estrutura da vegetação e, conseqüentemente, com a incidência de luz, das duas áreas estudadas. Da mesma forma, a estrutura da vegetação e a incidência luminosa podem também influenciar a estrutura etária das duas populações, fazendo com que estas ocupem em momentos diferentes as áreas, apresentando as diferenças encontradas.

\section{Agradecimentos}

Este estudo fez parte da disciplina Ecologia de Campo ministrada pelo Setor de Ecologia da Universidade do Estado do Rio de Janeiro, sendo parte dos resultados do Programa de Ecologia, Conservação e Manejo de Ecossistemas do Sudeste Brasileiro e do Projeto Vertebrados do Leste Brasileiro, Setor de Ecologia, Instituto de Biologia Roberto Alcântara Gomes, da Universidade do Estado do Rio de Janeiro. Os Autores agradecem aos professores da disciplina, pela infraestrutura, às Sub-reitorias de Graduação (SR-1) e de Pós-graduação (SR-2); à coordenação do Centro de Estudos Ambientais e de Desenvolvimento Sustentável (CEADS), pelo apoio dado à disciplina; à T.C. Rocha-Pessôa e E.M. Almeida, pelo auxílio na coleta de dados; à E.M. Almeida e M. Van Sluys, pela revisão do manuscrito oferecendo importantes sugestões. Durante o estudo R.D. Antonini recebeu bolsa de Iniciação Científica PIBIC/UERJ e A.F. Nunes-Freitas (processo CNPq 140722/2001-7) recebeu bolsa de doutorado.

\section{Referências bibliográficas}

Almeida, D.R.; Cogliatti-Carvalho, L. \& Rocha, C.F.D. 1998. As bromeliáceas da Mata Atlântica da Ilha Grande, RJ: composição e diversidade de espécies em três ambientes diferentes. Bromélia 5(1-4): 54-65.

Amézquita, P. 1998. Light environment affects seddling performance in Psychotria aubletiana (Rubiaceae), a tropical understory shrub. Biotropica 30: 126-129.
Araújo, D.S.D. \& Oliveira, R.R. 1988. Reserva Biológica Estadual da Praia do Sul (Ilha Grande, Estado do Rio de Janeiro): lista preliminar da flora. Acta Botanica Brasilica 1(2): 83-94.

Barnea, A.; Yom-Tov, Y. \& Friedman, J. 1992. Effect of frugivorous birds on seed dispersal and germination of multi-seeded fruits. Acta Ecologica 13(2): 209-219.

Brower, J.E. \& Zar, J.H. 1984. Field \& laboratory methods for general ecology. $2^{\text {nd }}$ ed. W.C. Brown Publishers, Iowa.

Canham, C.D.; Finzi, A.C.; Pacala, S.W. \& Burbank, D.H. 1994. Causes and consequences of resource heterogeneity in forests: interspecific variation in light transmission by canopy trees. Canadian Journal of Forest Reseach 24: 337-348.

Chazdon, R.L. 1986. Light variation and carbon gain in rain forest understory palms. Journal of Ecology 74: 995-1012.

Chazdon, R.L. \& Fetcher, N. 1984. Photosynthetic light environments in a lowland tropical rain forest in Costa Rica. Journal of Ecology 72: 553-564.

Clark, D.A. \& Clark, D.B. 1987. Temporal and environmental patterns of reproduction in Zamia akinneri, a tropical rain forest cycad. Journal of Ecology 75: 135-149.

Clark, D.B.; Clark, D.A.; Rich, P.M.; Weiss, S. \& Oberbauer, S.F. 1996. Landscape-scale evaluation of understory light and canopy structure: methods and application in a Neotropical lowland rain forest. Canadian Journal of Forest Research 26: 747-757.

Cogliatti-Carvalho, L. \& Rocha, C.F.D. 2001. Spatial distribution and preferential substrate of Neoregelia johannis (Carriére) L.B. Smith (Bromeliaceae) in a disturbed area of Atlantic Rainforest at Ilha Grande, RJ, Brazil. Revista Brasileira de Botânica 24(4): 389-394.

Costa, F.R.C. \& Magnusson, W.E. 2003. Effects of selective logging on the diversity and abundance of flowering and fruiting understory plants on a Central Amazonian Forest. Biotropica 35(1): 103-114.

Cunningham, S.A. 1997. The effects of light environment, leaf area, and stored carbohydrates on inflorescence production by a rain forest understory palm. Oecologia 111: $36-44$.

Denslow, J.S. 1987. Tropical rain forest and tree species diversity. Annual Review of Ecology and Systematic 18: 431-451.

Denslow, J.S.; Schultz, J.C.; Vitousek, P.M. \& Strain, B.R. 1990. Growth responses of tropical shrubs to treefall gap environments. Ecology 71(1): 165-179.

Dislich, R. \& Mantovani, W. 1998. A flora de epífitas da Reserva da Cidade Universitária "Armando de Salles Oliveira" (São Paulo, Brasil). Boletim Botânico da Universidade de São Paulo 17: 61-84.

Ellison, A.M.; Denslow, J.S.; Loiselle, B.A. \& Brénes, D.M. 1993. Seed and seedling ecology of Neotropical Melastomataceae. Ecology 74(6): 1733-1749.

Galetti, M. \& Stotz, D. 1996. Miconia hypoleuca (Melastomataceae) como espécie chave para aves frugívoras no Sudeste do Brasil. Revista Brasileira de Biologia 56(2): 435-439.

Hoffmann, J. 1998. Assessing the effects of environmental changes in a landscape by means of ecological characteristics of plant species. Landscape and Urban Planning 41: 239-248. 
Janzen, D.H. 1976. Why bamboos take so long to flower. Annual Review of Ecology and Systematics 7: 347-391.

Judd, W.S. 1986. Taxonomic studies in the Miconieae (Melastomataceae). I. Variation in inflorescence position. Brittonia 38(2): 150-161.

Levey, D.J. 1988. Tropical wet forest treefall gaps and distributions of understory birds and plants. Ecology 69: 1076-1089.

Levey, D.J. 1990. Habitat-dependent fruiting behaviour of an understory tree, Miconia centrodesma, and tropical treefall gaps as keystone habitats for frugivores in Costa Rica. Journal of Tropical Ecology 6: 409-420.

Lieberman, S.S. \& Dock, C.F. 1982. Analisys of the leaf litter arthropod fauna of a lowland tropical evergreen forest site (La Selva, Costa Rica). Revista de Biologia Tropical 30: $27-34$.

MacDougal, A. \& Kellman, M. 1992. The undestory light regime and patterns of tree seedlings in tropical riparian forest patches. Journal of Biogeography 19: 667-675.

Martins, F.R. 1993. Estrutura de uma floresta mesófila. $2^{\mathrm{a}}$ ed., UNICAMP.

Mesier, C. 1996. Managing light and understory vegetation in boreal and temperate broadleaf-conifer forest. Pp. 59-81. In: P.G. Comeau \& K.D. Thomas (eds.). Silviculture of temperate and boreal broadleaf-conifer mixtures. British Columbia Ministry of Forest, Victoria, B.C.

Meyer, J.Y. 1998. Observations on the reproductive biology of Miconia calvescens DC (Melastomataceae), an alien invasive tree on the Island of Tahiti (South Pacific Ocean). Biotropica 30(4): 609-624.

Oberbauer, S.F.; Clark, D.B.; Clark, D.A. \& Vega, G. 1993. Light environment, gas exchange, and annual growth of saplings three species of rain forest trees in Costa Rica. Journal of Tropical Ecology 9: 511-523.

Oliveira, R.R. 2002. Ação antrópica e resultantes sobre a estrutura e composição da Mata Atlântica da Ilha Grande, RJ. Rodriguésia 53(82): 33-58.
Pereira, T.S. \& Mantovani, W. 2001. Maturação e dispersão de Miconia cinnamomifolia (DC) Naud. na Reserva Biológica de Poço das Antas, município de Silva Jardim, RJ, Brasil. Acta Botânica Brasilica 15(3): 335-348.

Poole, R.W. 1974. An Introduction to Quantitative Ecology. McGraw-Hill, Inc., New York.

Poulin, B.; Wright, S.J.; Lefebvre, G. \& Calderón, O. 1999. Interspecific synchrony and asynchrony in the fruiting phenologies of congeneric bird-dispersed plants in Panama. Journal of Tropical Ecology 15: 213-227.

Rossi, L.B.M. \& Huguchi, N. 1998. Comparação entre métodos de análise do padrão espacial de oito espécies arbóreas de uma floresta tropical úmida. Pp. 41-59. In: C. Gascon \& P. Montinho (eds.). Floresta amazônica: dinâmica, regeneração e manejo. INPA.

Roy, J. 1990. In search of the characteristics of plant invaders. Pp. 335-352. In: D.I. Castri, F.; A.J. Hansen \& M. Debussche (eds.). Biological invasion in Europe and Mediterranean Basin. Kluwer Academic Publishers, Dordrecht, The Netherlands.

Schupp, E.W.; Howe, H.F.; Augspurger, C.K. \& Levey, D.J. 1989. Arrival and survival in tropical treefall gaps. Ecology 70(3): 562-564.

Snow, D.W. 1965. A possible selective factor in the evolution of fruiting seasons in Tropical Forests. Oikos 15: 274-281.

Stiles, F.G. \& Rosselli, L. 1983. Consumption of fruits of the Melastomataceae by birds: how diffuse is coevolution? Vegetatio 107/108: 57-75.

Svenning, J.C. 2000. Small canopy gaps influence plant distribution in the Rain forest understory. Biotropica 32(2): 252-261.

Veloso, H.P.; Rangek-Filho, A.L.R. \& Lima, J.C.A. 1991. Classificação da vegetação brasileira, adaptada a um sistema universal. Rio de Janeiro, Ed. IBGE. 123p.

Welden, C.W.; Hewett, S.W.; Hubbell, S.P. \& Foster, R.B. 1991. Sapling survival, growth, and recruitment: relationship to canopy height in a Neotropical forest. Ecology 72: 35-50.

Zar, J.H. 1999. Biostatistical analysis. $4^{\text {th }}$ ed. Prentice-Hall, New Jersey. 